\title{
High volume image-guided Injections for patellar tendinopathy: a combined retrospective and prospective case series
}

\author{
Sarah Morton 1 \\ Otto Chan ${ }^{2}$ \\ John King ${ }^{1,2}$ \\ David Perry ${ }^{1,2}$ \\ Tom Crisp ${ }^{1,2}$ \\ Nicola Maffulli2,3 \\ Dylan Morrissey ${ }^{1,2}$
}

1 Centre for Sports and Exercise Medicine, William Harvey Research Institute Barts and the London School of Medicine and Dentistry Queen Mary University of London, Mile End Hospital, London, UK

2 BMI London Independent Hospital

${ }^{3}$ Head of Department of Physical and Rehabilitation Medicine, University of Salerno, Azienda Ospedaliera San Giovanni di Dio e Ruggi D’Aragona, Salerno, Italy

\section{Corresponding author:}

Dylan Morrissey

Centre for Sports and Exercise Medicine

William Harvey Research Institute Barts and the London School of Medicine and Dentistry Queen Mary University of London, Mile End Hospital

Bancroft road

E1 4DG, London, UK

E-mail: d.morrissey@amul.ac.uk

\section{Summary}

Background: the aim was to quantify the effect of a novel high volume-image guided injection (HVIGI) technique for recalcitrant patellar tendinopathy (PT). Methods: twenty patients (8 prospective; 12 retrospective) with ultrasonographically confirmed proximal PT were recruited. A HVIGI under ultrasound guidance of $10 \mathrm{ml} 0.5 \%$ Bupivacaine, $25 \mathrm{mg}$ Hydrocortisone and $30 \mathrm{ml}$ normal saline at the interface of the patellar tendon and Hoffa's fat pad was administered. A standardised eccentric loading rehabilitation protocol was prescribed. Results: the VISA-P score improved from 45.0 to 64.0 (p<0.01) for all subjects, likely to be clinically significant. There was no statistically significant difference between the increase in the retrospective group of $19.9( \pm 23.5)$ and the prospective of $16.4( \pm 11.3) p=$ $0.7262 .5 \%$ of prospective subjects agreed that they had significantly improved, with $37.5 \%$ returning to sport within 12 weeks. Conclusions: HVIGI should be considered in the management of recalcitrant PT. Randomised controlled trials are warranted.

KEY WORDS: injection, patellar tendon, tendinopathy, ultrasound, VISA-P.

\section{Introduction}

Patients presenting with patellar tendinopathy (PT) will often describe a gradual onset and increase in symptoms with the symptoms typically described as a dull ache at the anterior-inferior knee that prevents activity ${ }^{1}$. It is a clinical diagnosis, with the VISA-P scale being a valid and reliable scale developed to assess severity ${ }^{2}$. The prevalence in elite athletes in jumping sports is estimated to be up to $14 \%$ at any given time, rising to $22 \%$ over a career, particularly in basketball and volleyball ${ }^{3}$. In contrast non-elite athletes have an estimated prevalence of $8.5 \%{ }^{4}$.

The precise aetiology and cause of pain remains unclear in tendinopathies. It had been accepted that painful overuse tendinopathies have a non-inflammatory pathology 5 . However a recently published work has suggested that chronic tendinopathy may be an active process of on-going tendon degeneration with many aspects of inflammation-mediated responses ${ }^{6}$. One proposed mechanism of tendon pathology includes three stages: reactive tendinopathy, tendon disrepair and degenerative tendinopathy ${ }^{7}$.

Ultrasonographic imaging of PT typically reveals decreased echogenicity, focal sonolucent regions (cysts) and intratendinous calcification ${ }^{1}$. Neovascular infiltration can be seen using Doppler ultrasound, and graded on ordinal scales ${ }^{7}$. The pain intensity of PT appears to be more related to the number of new vessels seen on Doppler ultrasound rather than the volume of blood in those vessels ${ }^{8}$.

Various treatments have been suggested for PT. Decline eccentric squat exercises are considered the primary line of treatment for PT, although outcomes remain poor with only an estimated $55 \%$ returning to good or excellent status over long periods of time 9 . Heavy slow resistance training has been shown to be equivalent to eccentric training ${ }^{9}$. Extracorporeal shock wave therapy has shown mixed benefits and so its role is yet to be clearly established ${ }^{9}$. It is suggested that there is no difference between injection and control groups when using platelet-rich plasma injections $^{9}$. There is also mixed evidence on sclerosing in- 
jections ${ }^{9}$. There is no supportive evidence for the use of steroid injections, autologous blood and dry needling ${ }^{9}$. Surgery does appear to improve VISA-P scores but is comparable to conservative eccentric exercises, yet requires a long period of rehabilitation ${ }^{9}$.

High volume image guided injections (HVIGI) in Achilles tendinopathy have been shown to significantly reduce pain and improve function, measured using the VISA-A score ${ }^{10}$. The hypothesised mechanism behind the success of the injection is believed to be a local mechanical effect of the injection stretching, breaking or occluding neo-vessels and the accompanying nerve supply, which is believed to mediate tendinopathic pain ${ }^{10}$. The HVIGI must then be followed with a physiotherapist led eccentric rehabilitation programme designed to progressively load and therefore remodel the tendon, promote muscle function recovery and therefore enable return to optimal activity.

A small retrospective pilot study was carried out in 2008 into the effect of a high volume injection on patellar tendinopathy with promising results ${ }^{11}$. The aim of our study was to establish the effect of HVIGIs on patellar tendinopathy, including the use of prospective data, especially as the anatomical and biomechanics differs from that of the Achilles tendon. The objective was to complement the original retrospective data from the pilot study with additional retrospective and prospective data, thus allowing followup without recall bias.

\section{Materials and methods}

\section{Subjects}

All subjects who attended for a HVIGI for proximal PT between January 2012 and April 2012 at a specialist centre were recruited prospectively, resulting in a total of eight subjects. They had all failed conservative management (i.e. at least 3 months of decline eccentric squats) and had undergone a range of other therapies up to that point including massage, steroid injections, acupuncture, bracing and ultrasound. Due to presentation stage, our patients would have been at the degenerative stage of tendinoapthy which involves areas of cell death, disordered matrix, neovascularisation and disordered collagen, with limited capacity for reversal of these changes ${ }^{7}$. The questionnaire was administered prior to the HVIGI and at 12 weeks post-injection. Twelve patients were recruited retrospectively over two time periods from the same specialist centre; nine of these were from the original pilot study and the remaining three were recruited from May 2011 to November 2011. Again all had failed conservative management. The questionnaire was sent at a mean of 9 months following the treatment.

The subjects recruited were aged between 18 and 65. The clinical diagnosis by a sports physician, including both pain and stiffness, of patellar tendinopathy was confirmed with ultrasound imaging of the patellar tendon showing neovascularisation on Doppler Ultrasound. Patients were excluded if they had ever had previous patellar tendon surgery or a complete or partial tear of the patellar tendon.

\section{Procedure}

The diagnosis of patellar tendinopathy was confirmed using ultrasound with a note made of tendon size, the neovascularisation grade and the presence of cysts, bursae or calcification (Fig. 1). All ultrasound scanning and interventions were carried out by a single experienced radiologist using the same ultrasound scanner throughout (Elegra; Siemens, Erlangen, Germany). A $13 \mathrm{MHz}$ transducer was used alongside power Doppler imaging. The patellar tendon thickness was measured in the longitudinal direction ${ }^{12}$. Neovascularisation was graded using a Modified Ohberg scale ${ }^{13}$.

The patients were injected using an aseptic technique under real-time ultrasound guidance with $10 \mathrm{ml}$ of $0.5 \%$ Bupivacaine mixed with $25 \mathrm{mg}$ of hydrocortisone followed by $30 \mathrm{ml}$ of normal saline in the prospective patients at the interface between the tendon and Hoffa's fat pad using a $21 \mathrm{G}$ green needle, adjacent to the area of neovascularisation. The volumes were administered using $10 \mathrm{ml}$ syringes one after another to maintain the high pressure as quickly as the pressure allowed. The knee was at a $90^{\circ}$ angle for injection and the needle could be moved using the ultrasound guidance to ensure the areas of maximal neovascularisation were targeted. In the retrospective patients there was a range of saline volumes used, of up to $40 \mathrm{ml}$, due to the data being from earlier on in the development of the procedure. No injectate was administered directly into the tendon (Fig. 2).

Subjects were advised to take ibuprofen three times a day for two days to allow for any post-injection flare in pain. Following the injections all subjects were asked to follow the same rehabilitation programme as advised by a chartered physiotherapist. Initially there was three days relative rest with daily life activities only. On Day 4 decline eccentric squat loading was re-started with patients made aware of a potential reactive response. From Day 7 non-impact cardiovascular exercise was re-introduced with sport from Day 12 at the earliest, depending on pre-injection status and the amount of time away from sport.

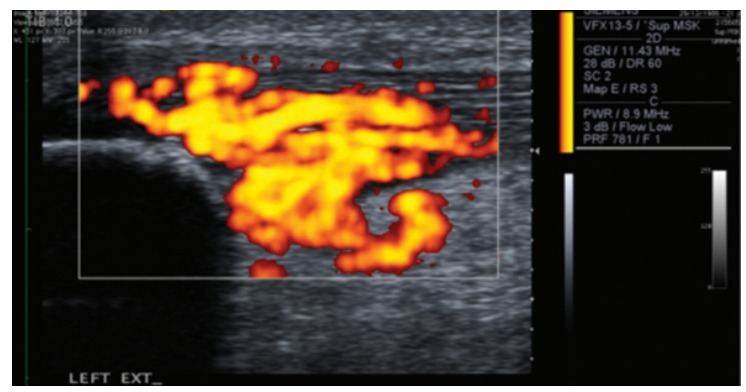

Figure 1. Ultrasound image showing Doppler imaging of neovascularisation of proximal patellar tendinopathy with tendon relaxed prior to HVIGI. 


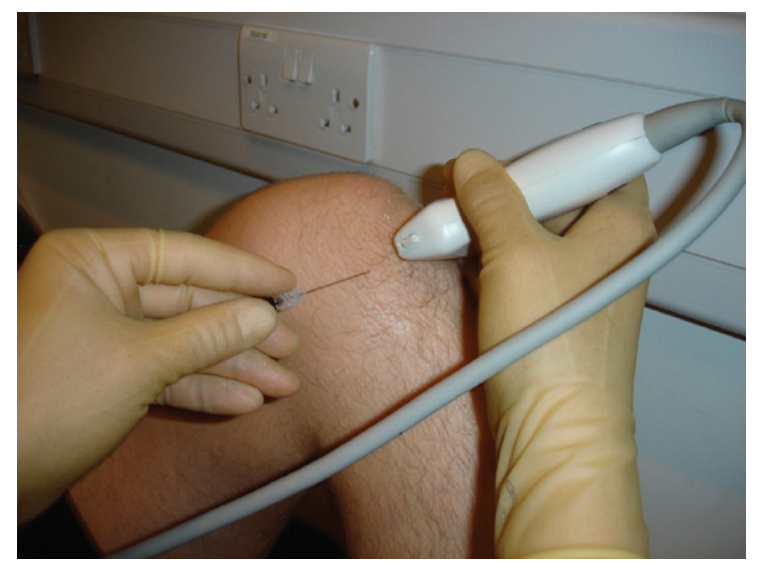

Figure 2. High Volume Image Guided Injection of Proximal Patellar Tendinopathy.

\section{Outcome measure}

Prospective subjects were asked to complete an online questionnaire on the day of presentation which included the VISA-P. The VISA-P score has been documented as a reliable assessment of recovery from patellar tendinopathy, with a score of 100 representing a fully functional knee ${ }^{2}$. An e-mail reminder was then sent at 12 weeks following treatment to ask them to repeat the questionnaire. The change in the VISA-P score was considered the main outcome. Three Likert Scales were also included asking whether the subjects agreed their symptoms had improved, their strength had improved and whether they had returned to the level of sport they wanted to. Subjects were also able to comment on any additional treatment they had received in that 12 week period outside of the physiotherapy rehabilitation programme and on any adverse effects encountered.

Retrospective subjects were either sent two paper copies of the questionnaire (original pilot study) or a link for the email version (subjects from 2011). They were asked to complete one questionnaire as though they were filling it in immediately prior to the injection and one for their current status, giving a mean follow up period of 9 months.
Data was collected from the ultrasound reports regarding the mean tendon size and the neovascularisation grade at the time of injection. The presence of cysts, calcium or bursae was also noted from the ultrasound reports.

\section{Analysis}

All subjects were allocated a code to ensure confidentiality and anonymity. VISA-P scores were added to provide a total. Descriptive statistics were calculated. The Wilcoxon Signed Ranks test was used to analyse the prospective and retrospective data using SPSS (v.20, IBM, USA). Significance was set at $p<0.05$. A sub-group analysis was carried out of subjects with an initial VISA-P of less than 40 compared to greater than 40 using the Wilcoxon Signed Ranks test. The change in VISA-P score between the retrospective and prospective subjects was also compared using the Wilcoxon Signed Ranks test.

\section{Ethical Considerations}

Ethical approval was obtained from Queen Mary's University of London Ethics and Research Committee, with consent obtained via the questionnaire.

Standards and laws were adhered to as for Padulo et al.'s editorial ${ }^{14}$.

\section{Results}

\section{Subject characteristics}

The subject characteristics of the twenty subjects followed prospectively are shown in Table 1.

\section{Ultrasound Findings}

Table 2 shows the ultrasound findings of the eight subjects followed prospectively at initial presentation andthe ultrasound information available for the retrospective subjects recruited from 2011. Detailed information for the retrospective subjects from the original

Table 1. Subject Characteristics.

\begin{tabular}{lll}
\hline Characteristic & Retrospective & Prospective \\
\hline Age & $30.8 \pm 8.0$ & $32.4 \pm 6.3$ \\
\hline Gender & 11 male: 1 female & 8 male: 0 female \\
\hline Length of symptoms prior to injection (months) & $18.9 \pm 10.9$ & \\
& {$[3$ data items missing] } & $20.8 \pm 13.8$ \\
\hline Weekly hours of sport & Data unavailable & $9.4 \pm 4.2$ \\
Level of sport & 4 Senior professional & 1 Senior professional \\
& 2 Semi-professional & 2 Semi-professional \\
& 3 Competitive amateur & 4 Occasional amateur competition \\
& 1 Fun/fitness & 1 Fun/fitness \\
& 1 Other & [2 data items unavailable] \\
\hline
\end{tabular}


pilot study was unavailable. Following the injection there was instantaneous resolution of the neovascularisation in all subjects on ultrasound.

\section{VISA-P findings}

The Wilcoxon Signed Ranks Test showed a significant change in the mean VISA-P score for the combined retrospective and prospective data of 18.5 ( $Z=-$ $3.62, p<0.01$ ) with a pre-injection mean of $45.5 \pm 20.1$ and post-injection $64.0 \pm 19.9$.

The Wilcoxon Signed Ranks Test also showed a significant change in the VISA-P score for both the prospective and retrospective data when analysed separately. The prospective data showed a change in the mean VISA-P from $41.5 \pm 13.3$ to $57.9 \pm 17.4$, a change of 16.4 over 12 weeks $(Z=-2.52, p=0.01)$. The retrospective data showed a change in the mean VISA-P from $48.1 \pm 23.7$ to $68.0 \pm 21.1$, a change of 19.9 over a mean follow-up of 9 months $(Z=-2.66, p<0.01)$. There was no statistically significant difference in the mean change in VISA-P between a follow up of 12 weeks and a follow up of 9 months $(Z=-0.351, p=0.73)$.
Figure 3 shows the results of each individual VISA-P score before and after the injection. All prospective subjects showed some improvement in symptoms (Fig. 3A shows prospective subjects; Fig. 3B shows retrospective subjects).

\section{VISA-P sub-analysis}

A sub-analysis was carried out using the Wilcoxon Signed Ranks Test on the mean change in VISA-P where the pre-HVIGI VISA-P score was less than 40 in comparison to the mean change in VISA-P score when the pre-HVIGI VISA-P score was greater than 40 in order to assess whether subjects with higher initial severity demonstrated particular patterns of improvement (Tab. 3).

There was no statistically significant difference $(Z=$ $-1.25, p=0.21$ ) between the mean improvement when the initial VISA-P was less than 40 when compared to the mean improvement when the initial VISA-P is greater than 40 , although it is sometimes clinically apparent that patients with more severe presentations have the greatest benefit.

Table 2. Ultrasound findings.

\begin{tabular}{llll}
\hline Characteristic & Retrospective & Prospective & $\boldsymbol{p}$ value \\
\hline Leg injected & 2 right: 1 left & 5 right: 3 left & - \\
\hline Mean Size of Tendon on US scan $(\mathrm{mm})$ & $11.3 \pm 0.7$ & $10.3 \pm 2.5$ & 0.31 \\
\hline Mean Neovascularisation Grade & $3.0 \pm 1.0$ & $3.75 \pm 0.7$ & 0.31 \\
\hline Scans with cysts presence & 0 & 2 & - \\
\hline Scans with calcium present & 0 & 1 & - \\
\hline Scans with bursae present & 1 & 0 & - \\
\hline
\end{tabular}
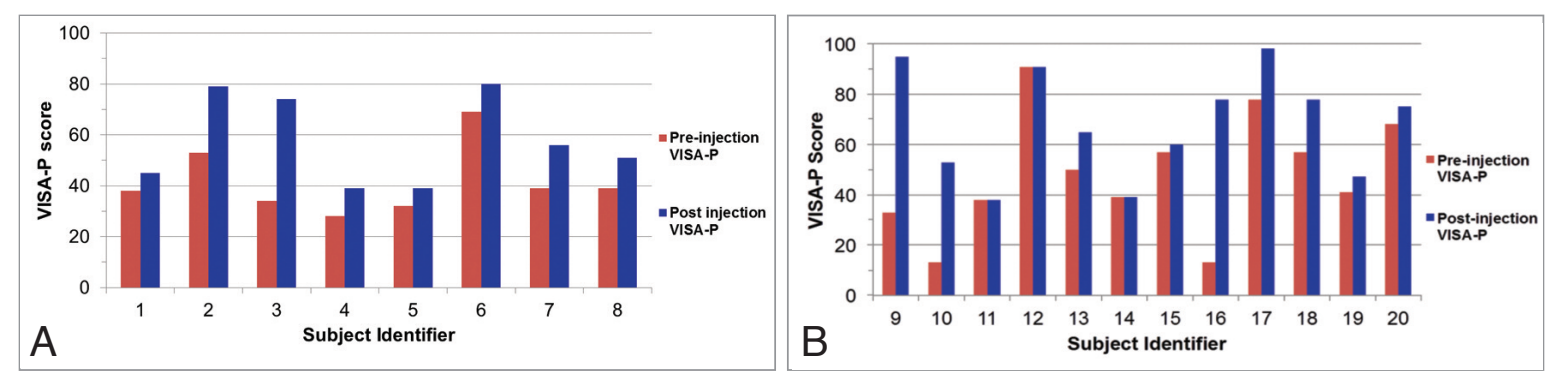

Figure 3 A-B. (A) The VISA-P score before and 12 weeks after the HVIGI for each individual subject followed prospectively. (B) The VISA-P score before and after the HVIGI for each individual subject followed retrospectively over a mean of 9 months.

Table 3. Comparison of Mean Change in VISA-P when initial VISA-P less than 40 compared to more than 40.

\begin{tabular}{llll}
\hline & Group Population & Pre-HVIGI Mean VISA-P $( \pm$ SD) & Mean Change in VISA-P ( \pm SD) \\
\hline Initial VISA-P $<40$ & 11 & $32.4( \pm 9.9)$ & $23.7( \pm 23.9)$ \\
\hline Initial VISA-P $>40$ & 9 & $62.7( \pm 15.4)$ & $12.1( \pm 8.9)$ \\
\hline
\end{tabular}




\section{Prospective subjects rating of treatment}

Of the eight subjects followed prospectively, five either agreed or strongly agreed that their symptoms had improved in the 12 weeks following the injection along with their strength. One subject strongly agreed they had been able to return to the level of sport they wanted to play at, with two agreeing they were approaching the level at which they wanted to compete. One subject stated they had returned to full training before a medial ligament tear prevented them from playing sport. One subject reported they felt they had returned to sport too soon and had a repeat HVIGI eleven weeks following their initial injection. No subjects reported a flare up in pain post injection, although the compliance to the suggested ibuprofen following the injection is also unknown.

\section{Discussion}

\section{Principal findings}

This is the first study of high volume image-guided injections that includes the use of prospective data. The majority of subjects included showed some improvement in their VISA-P score, with a mean improvement of $18.5 \pm 19.2$, therefore being clinically as well as statistically significantly different. No deterioration following a HVIGI was shown, allaying concerns about tissue damage at least in the short and medium term.

\section{Strength and weakness of the study and compari- son to other studies}

Of the prospective subjects, $62.5 \%$ either agreed or strongly agreed their symptoms and strength had improved, which is comparable to the estimated 55\% who return to good or excellent using eccentric training alone ${ }^{9}$. It should also be noted that these patients had already failed conservative management, and therefore are part of the $45 \%$ who do not return to good or excellent using eccentric or heavy slow resistance protocols, and additionally reported a long symptom duration (mean symptom duration $=19.7 \pm$ 12.0). This is also comparable to open surgery on the patellar tendon where $74 \%$ returned to good or excellent, although return to sport time is often longer, typically averaging at least 7 months ${ }^{15,16}$. Arthroscopic surgical techniques are considered equivalent to open surgical techniques in patellar tendinopathy ${ }^{17}$. There is also additional risk of complications in surgery with early complications including wound haematoma and superficial infection and late complications including anterior knee pain on kneeling ${ }^{16}$.

No adverse outcomes were reported in the HVIGIs in this study, possibly because image guidance enables diagnostic confirmation and ensures needle placement is precise ${ }^{18}$. During the HVIGI the separation of the tendon from the underlying tissue can be visu- alised on ultrasound guidance. The use of Power Doppler also enables the radiologist to visualise the tissue disruption of the neovascularisation immediately following the injection. This is likely to be comparable to the use of arthroscopic shaving of the dorsal side of the proximal patellar tendon to remove the neovascularisation, without the side-effects of surgery such as the use of crutches and slower rehab ${ }^{19}$. Therefore, to have $37.5 \%$ of patients return to the level of sport they want to play at within 12 weeks is an indication of the benefits of this treatment. However, what remains unclear is whether the chemical effect of both the hydrocortisone and/or the Bupivacaine is also required, or whether it is purely the mechanical effect of the large volumes injected stretching or stripping the neovascularisation and this therefore requires additional research. We also recognise that the use of corticosteroids in tendinopathy has been shown to be beneficial in the short term but not in the long term for tendinopathy ${ }^{20}$. We therefore believe that it is not only the HVIGI but the standardised eccentric rehabilitation programme that equates to the results seen; if the HVIGI allows the patient to complete an eccentric programme in the short term this is likely to have long term gains.

Unlike the prospective subjects, some of the retrospective subjects did not show any improvement in their VISA-P scores over nine months. It would therefore be interesting to continue the follow-up of the prospective patients to nine months to compare outcomes at this stage to see if there is any decline in function. It is unclear why some patients respond much better than others, as demonstrated by the range of 0 to 65 point improvement on the VISA-P scores of the retrospective subjects, which may suggest some individuals regress after the three month stage. Whilst the retrospective data adds to the numbers, and therefore power, of the study it is recognised that the retrospective introduces recall bias. It has however allowed some indication of the patients' trajectories over a longer time period.

The sub-analysis comparing the improvement of those with an initial VISA-P score of less than 40 to the improvement of those with an initial VISA-P score greater than 40 , although not statistically significant, does show a trend towards significance. There was a $23.7 \pm 23.9$ improvement in the less than 40 group in comparison to the $12.1 \pm 8.9$ in the greater to 40 group. As mentioned above a VISA-P improvement of more than 20 is considered clinically significant and it may therefore be that those with more severe patellar tendinopathy actually respond better to a HVIGI than those who are less severe. This is promising as often it is the patients with the more severe condition that are harder to treat and so larger studies are now indicated. Compliance to the rehabilitation programme was not assessed and may be a key factor in that the purpose of the injection is to enable the rehabilitation to take effect. A VAS pain scale may therefore have been useful to include as a complement to the VISA$P$, especially as VAS pain scales are well validated in patellar tendinopathy ${ }^{21}$. 


\section{Implications}

High volume image-guided injections should be considered as a treatment in recalcitrant chronic patellar tendinopathy, when conservative measures are not yielding an optimal result. The findings are comparable to other treatment modalities despite including patients with a long symptom duration of 19.7 months, who had already failed conservative management.

\section{Future research}

The gold standard research for the future would be a randomised control trial with sufficient power to establish the benefits of HVIGI in patellar tendinopathy when compared to other conservative treatments, such as eccentric exercise. Sub-group analysis would also determine the features which predict a positive result from treatment. It is important to ensure that side effects are monitored over a prolonged period, and it would be ideal to explore the therapeutic mechanisms under pinning the clinical effects seen, including rescanning the tendon to note any change in tendon size or neovascularisation. As mentioned above, it is also important to establish the mechanism of action of the HVIGI, including whether the administration of hydrocortisone and/or Bupivacaine is required.

It would also be of benefit to consider the effects of other treatments either in conjunction with or prior to HVIGIs. Rehabilitation experience and compliance following HVIGI must also be standardised - as in this study - and measured in the follow up phases. It may be that compliance to the post-HVIGI regime therefore affects outcomes. Although not studied it is also known that some patients have repeat HVIGIs after a period of time, which anecdotally appears to be of benefit, although this needs confirming. Qualitative experience of the patient's experience of HVIGls would be useful so that comparisons can be made, for example, to surgery and shockwave therapy, to see which is more acceptable in terms of rehabilitation.

\section{References}

1. Khan KM, Maffulli N, Doleman BD, Cook JL, Taunton JE. Patellar tendinopathy: some aspects of basic science and clinical management. Br J Sports Med. 1998;32:346-355.

2. Visentini PJ, Khan KM, Cook JL, Kiss ZS, Harcourt PR, Wark JD. The VISA score: an index of severity of symptoms in patients with jumper's knee (patellar tendinosis). Victorian Institute of Sport Tendon Study Group. J Sci Med Sport. 1998; 1:22-28.

3. Lian OB, Engebretsen L, Bahr R. Prevalence of jumper's knee among elite athletes from different sports - A cross-sectional study. Am J Sports Med. 2005;33:561-567.

4. Zwerver J, Bredeweg SW, van den Akker-Scheek I. Preva- lence of Jumper's Knee Among Nonelite Athletes From Different Sports A Cross-Sectional Survey. Am J Sports Med. 2011;39:1984-1988.

5. Khan KM, Cook JL, Kannus P, Maffulli N, Bonar SF. Time to abandon the "tendinitis" myth - Painful, overuse tendon conditions have a non-inflammatory pathology. BMJ. 2002;324: 626-627.

6. Rees JD, Stride M, Scott A. Tendons: time to revisit inflammation? Br J Sports Med. 2013;47:e2.

7. Cook JL, Purdam CR. Is tendon pathology a continuum? A pathology model to explain the clinical presentation of load-induced tendinopathy. Br J Sports Med. 2009;43:409-416.

8. Cook JL, Malliaras P, De Luca J, Ptasznik R, Morris M. Vascularity and pain in the patellar tendon of adult jumping athletes: a 5 month longitudinal study. Br J Sports Med. 2005;39:458461.

9. Gaida JE, Cook J. Treatment Options for Patellar Tendinopathy: Critical Review. Curr S;ports Med Rep. 2011;10:255-270.

10. Chan O, O'Dowd D, Padhiar N, et al. High volume image guided injections in chronic Achilles tendinopathy. Disabil Rehabil. 2008:30:1697-1708.

11. Crisp T, Khan F, Padhiar N, et al. High volume ultrasound guided injections at the interface between the patellar tendon and Hoffa's body are effective in chronic patellar tendinopathy: A pilot study. Disability Rehabil. 2008;30:1625-1634.

12. Fredberg U, Bolvig L, Andersen NT, Stengaard-Pedersen K. Ultrasonography in evaluation of Achilles and patella tendon thickness. Ultraschall Med. 2008;29:60-65.

13. Del Buono A, Chan O, Maffulli N. Achilles tendon: functional anatomy and novel emerging models of imaging classification. Int Orthop. 2013;37:715-721.

14. Padulo J, Oliva F, Frizziero A, Maffulli N. Muscles, Ligaments and Tendons Journal. Basic principles and recommendations in clinical and field science research. MLTJ. 2013;4:250-252.

15. Rodriguez-Merchan EC. The treatment of patellar tendinopathy. J Orthop Traumatol. 2013;14:77-81.

16. Maffulli N, Binfield PM, Leach WJ, King JB. Surgical management of tendinopathy of the main body of the patellar tendon in athletes. Clin J Sports Med. 1999;9:58-62.

17. Muccioli GMM, Zaffagnini S, Tsapralis K, Alessandrini E, Bonanzinga $T$, Grassi $A$, et al. Open versus arthroscopic surgical treatment of chronic proximal patellar tendinopathy. A systematic review. Knee Surg Sports Traumatol Arthrosc. 2013;21: 351-357.

18. Fredberg U, Bolvig L, Pfeiffer-Jensen M, Clemmensen D, Jakobsen BW, Stengaard-Pedersen K. Ultrasonography as a tool for diagnosis, guidance of local steroid injection and, together with pressure algometry, monitoring of the treatment of athletes with chronic jumper's knee and Achilles tendinitis: a randomized, double-blind, placebo-controlled study. Scand J Rheumatol. 2004;33:94-101.

19. Willberg L, Sunding K, Ohberg L, Forssblad M, Alfredson H. Treatment of Jumper's knee: promising short-term results in a pilot study using a new arthroscopic approach based on imaging findings. Knee Surg Sports Traumatol Arthrosc. 2007;15:676-681.

20. Coombes BK, Bisset L, Vicenzino B. Efficacy and safety of corticosteroid injections and other injections for management of tendinopathy: a systematic review of randomised controlled trials. Lancet. 2010;376:1751-1767.

21. Khan KM, Visentini PJ, Kiss ZS, et al. Correlation of ultrasound and magnetic resonance imaging with clinical outcome after patellar tenotomy: prospective and retrospective studies. Victorian Institute of Sport Tendon Study Group. Clin J Sport Med. 1999;9:129-137. 